\title{
The Dust Content and Temperature Distribution of Galaxies in The Local Universe
}

\author{
S. Falony ${ }^{1}$, M. Baes ${ }^{1}$, J. I. Davies ${ }^{2}$, and C. Vlahakis ${ }^{3}$ \\ ${ }^{1}$ Universiteit Gent, Belgium, email: sarah.falony@ugent.be \\ ${ }^{2}$ Cardiff University, UK \\ ${ }^{3}$ Bonn University, Germany
}

\begin{abstract}
We measured the SCUBA fluxes of a sample of Seyfert 1, Seyfert 2, starburst and normal disc galaxies, taken from the Extended 12 Micron Galaxy Sample. From these data, we determine the entire dust content and temperature distribution of galaxies of different classes through a Planckian inversion technique.
\end{abstract}

The best way to determine the dust content of galaxies is by measuring their emission in the FIR and submm regions. Unfortunately, there is a strong degeneracy between dust mass and temperature; in order to determine the amount and temperature distribution of dust in a reliable way, a good spectral coverage of the entire FIR-submm SED is necessary. We use a data set extracted from the Extended 12 Micron Galaxy Sample, for which the spectral energy distribution has been measured out to $200 \mu \mathrm{m}$ with IRAS and ISO. This sample consists of representative subsamples of Seyfert 1, Seyfert 2, starburst and normal disc galaxies. We extended some of the SEDs with additional 450 and $850 \mu \mathrm{m}$ fluxes, measured with SCUBA on the JCMT. We apply a direct inversion technique to determine the dust mass and temperature distribution, based upon an inverse Planckian transform technique. We compared these models to models with a 2-component modified blackbody fit. We find that submm data are particularly important for constraining the dust content and temperature distribution in these galaxies, as some very cold dust can remain hidden from the FIR data alone.
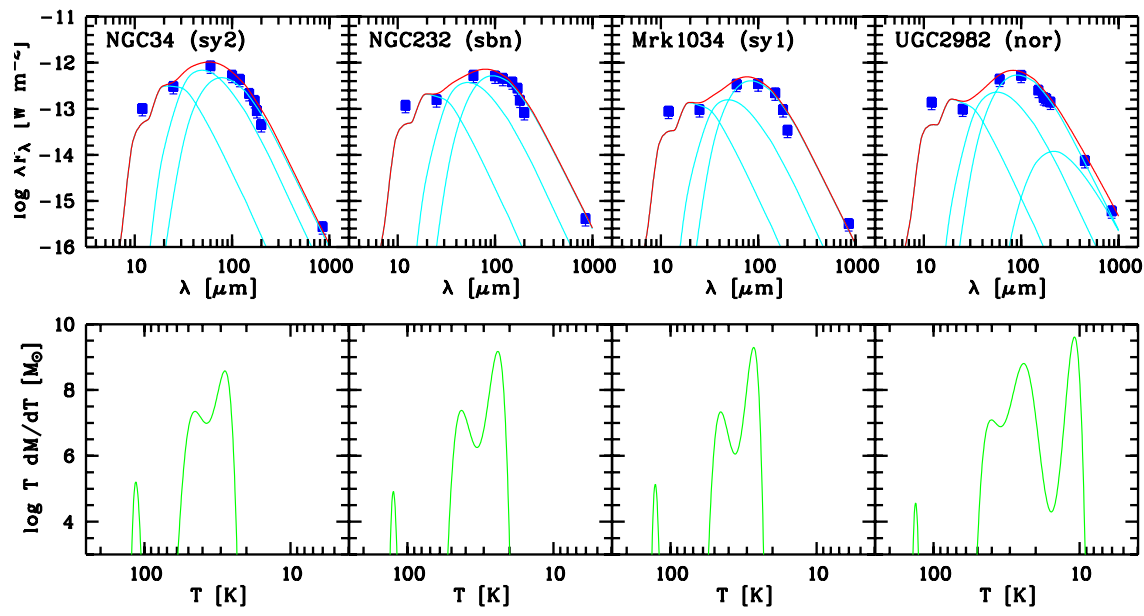

Figure 1. SED decomposition (upper panels) and dust temperature distribution (bottom panels) resulting from the direct inversion technique applied to four galaxies in our sample. 